\title{
LA RECEPCIÓN DE LAS NOTICIAS DE LA GUERRA DE LA INDEPENDENCIA EN CUBA Y LA REACCIÓN DE LOS CIUDADANOS (1807-1812)
}

\author{
Sigfrido Vázquez Cienfuegos \\ Universidad de Sevilla/ Universidad Económica de Praga (VŠE)
}

\section{RESUMEN}

La llegada de noticias a la isla de Cuba sobre lo que ocurrió en la Península durante la Guerra de la Independencia fue posible, a pesar de las dificultades en las comunicaciones oficiales por el dominio británico del mar, gracias a la puesta en práctica de todo un servicio de información por parte del capitán general de La Habana, marqués de Someruelos, que obtuvo noticias fehacientes desde los Estados Unidos. La llegada de prensa desde Europa, rumores y comunicaciones privadas al puerto habanero, así como la posterior publicación de noticias en la propia prenda cubana, permitió a la población cubana tener conocimiento de los movimientos políticos acontecidos al otro lado del Océano y a actuar en consecuencia de los mismos.

Palabras clave: La Habana, Someruelos, libertad de prensa, abolicionismo, Constitución 1812.

\section{ABSTRACT}

The arrival of news to the island of Cuba about Spain during the Peninsular War was possible, despite the difficulties in official communications by British sea control, for the implementation of an information service of reliable news from the United States of America by the captain general of Havana, marquis of Someruelos. The arrival of press from Europe, rumours and private communications to the Havana harbour and the subsequent publication of news in Cuba allowed the Cuban people have knowledge about the politic situation in Spain.

Key words: Havana, Someruelos, press freedom, abolition, 1812 Spanish Constitution.

\footnotetext{
1 Este trabajo está enmarcado en el proyecto de investigación "El municipio indiano: relaciones interétnicas, económicas y sociales (HUM 468)" del grupo de investigaciớn "Andalucía y América: Tierra y sociedad (HUM 292)" de la Universidad de Sevilla.
} 


\section{Introducción}

Para comenzar quiero explicar cual es la razón que me ha llevado a decidir los límites temporales para este trabajo. La fecha de inicio la he situado algo antes de lo que viene siendo la norma para fijar el comienzo de la Guerra de la Independencia, con los acontecimientos del 2 de mayo.

Me parece que circunscribir el inicio del periodo estudiado a esta fecha, por mucho que ahora estemos en la conmemoración de su bicentenario, implica una limitación que puede estar impidiendo el estudio de las causas más inmediatas que desencadenaron el conflicto que ahora nos ocupa. Desde un punto de vista puramente militar, seguir situando el inicio del hecho de la Guerra en mayo de 1808 me parece poco acertado, y desde una perspectiva político-jurídica, tampoco tiene mayor sentido. Esta persistencia en mantener la fecha del 2 de mayo como referencia solamente demuestra la fuerza del mito en nuestra historiografía.

Desde el punto de vista político dos hechos acontecidos en 1807 fueron determinantes y decisivos en el devenir de los acontecimientos del año siguiente. En primer lugar, la erección del Almirantazgo por Manuel Godoy supuso la culminación de las aspiraciones del Favorito, pero a su vez irritó a todos, a uno y otro lado del Atlántico, pues por una parte el cargo implicó el tratamiento de Alteza Serenísima, algo que acabó de soliviantar al príncipe de Asturias y los que en torno a él se unieron contra Godoy, pero del mismo modo significó un nuevo planteamiento comercial, que molestó en toda América. En segundo lugar, la consecuencia directa de esta decisión política fue la Conjura de El Escorial, bochornoso asunto en la que el rey llega a proclamar nada menos que el príncipe Fernando, sucesor en el trono de Carlos IV, tuvo la intención de acceder a la corona por medio de lo que hoy nadie dudaría en calificar de golpe de Estado. Esta conjura fue el antecedente de lo que en marzo de 1808 sucedería en Aranjuez.

En el plano militar parece que fue mucho más importante que los sucesos de Madrid de 2 de mayo de 1808 la firma del Tratado de Fontainebleau de 1807, llevada a cabo el mismo mes de octubre en que tuvo lugar el suceso de El Escorial. Fontainebleau fue la claudicación ante los deseos de Napoleón, casi una capitulación de hecho que permitió que las tropas francesas iniciasen la ocupación de la Península. No creo que pueda dudarse de que este despliegue de tropas extranjeras fue un hecho bélico violento con la ocupación de varias plazas españolas, contrario a la legalidad establecida en el tratado, al no respetar siquiera el mismo acuerdo, y que posteriormente fue determinante en el desarrollo de los momentos iniciales de la contienda durante el año 1808.

Que las autoridades de la época no supiesen comprender el alcance de los acontecimientos de 1807 o que fuesen incapaces de tomar medidas al respecto es una cuestión que no debería impedir la justa valoración histórica, que creo que debería situar el inicio del proceso histórico que examinamos en dicho año. A pesar de estos hechos, conocidos por la historiografía, parece seguir pesando más el momento "heroico" del 2 de mayo que no creo que diera lugar a cuestiones determinantes de carácter estratégico o militares para uno u otro bando, y si sirvió políticamente fue como primer ejemplo patriótico-propagandístico de la resistencia frente al francés.

He llegado a este convencimiento de que había que situar el inicio de la Guerra de la Independencia en octubre de 1807 no por un capricho de joven historiador con deseos iconoclastas, sino porque al estudiar la historia de Cuba durante este periodo, estos hechos aparecen como fundamentales para entender el devenir posterior de la situación en Cuba y creo que de toda América. En definitiva, de toda la Historia de España.

Desde finales del siglo XVIII, en los elementos de la disputa política había aparecido un factor totalmente externo a la situación habanera y este era la vinculación o no a Manuel Godoy de los mandos políticos y militares de la isla. Esta cuestión venía siendo desde 1801 determinante para el desarrollo de las distintas políticas en Cuba, pero especialmente en cuestiones de carácter económico. No voy a profundizar aquí en este asunto, ${ }^{2}$ pero si señalaré básicamente qué implicó para Cuba la adscripción o no de sus regidores al bando godoyista. De una manera escueta y algo simplista, los jefes de la

Sí lo hago en el libro que está próxima su publicación Tan difíciles tiempos para Cuba. El gobierno del marqués de Someruelos (1799-1812). 
Intendencia y la Comandancia de Marina de La Habana se habían destacado como defensores de los derechos monopolísticos y de las prebendas especiales otorgadas por el príncipe de la $\mathrm{Paz}$, mientras que el Gobierno y Capitanía General se había posicionado junto a los poderes locales (Cabildo y Consulado) en la defensa de los derechos e intereses de los habaneros.

El hecho que vino a colmar el vaso de las rivalidades y enfrentamientos fue la instauración del Almirantazgo por Manuel Godoy, celebrado en La Habana en septiembre de 1807. Este asunto, casi ignorado hasta ahora, al menos para el caso cubano, fue el detonante para crear un malestar general en La Habana contra las autoridades peninsulares, las cuales eran identificadas en la isla con los intereses de Godoy. A fines de año llegaron las noticias sobre los sucesos acaecidos en El Escorial en octubre. En la celebración del suceso en enero de 1808 por parte de los godoyistas, volvieron a plantearse las discrepancias políticas que sacudían la capital cubana. ${ }^{3}$ Como puede observarse las tensiones políticas estaban planteadas en Cuba mucho antes de que los sucesos de mayo sacudiesen toda la monarquía hispánica.

El límite final del periodo para este estudio, se puede situar en la llegada de la Constitución de 1812 a La Habana. Si bien, el conflicto bélico siguió su curso en la Península, las repercusiones de las decisiones tomadas en Cádiz durante los años anteriores habían permitido a los cubanos unos logros, principalmente económicos, que hicieron que sus reivindicaciones hubiesen quedado colmadas cuando la Carta Magna fue jurada en La Habana. Por ello he considerado que era mucho más interesante centrar la atención sobre los años entre 1807 y 1812.

\section{Llegada de noticias a la isla}

Para el estudio de la recepción de noticias en la isla de Cuba en este periodo hay que tener en cuenta varios condicionantes sin los cuales no se comprendería la particularidad e importancia del tema.

\section{Comunicaciones oficiales}

En primer lugar el condicionante más obvio es la insularidad del territorio, lo que implicó no pocas limitaciones. Cuba sólo podía recibir órdenes y noticias por vía marítima, un hecho que no es trivial. En el momento histórico que estamos analizando había significado toda una serie de limitaciones hasta 1808 por el dominio británico del mar. Las comunicaciones oficiales desde el reinicio de la guerra contra Gran Bretaña en 1804 se habían entorpecido de tal modo que apenas si llegaron órdenes en los años anteriores a los sucesos desencadenados entre 1807 y 1808 .

Las autoridades cubanas daban cuenta de los continuos apresamientos de los correos oficiales por buques de guerra ingleses o por parte de corsarios franceses. El gobernador y capitán general de La Habana, marqués de Someruelos, tuvo que dar cuenta en enero de 1806 de que la última correspondencia de oficio había llegado a en abril de $1805 .{ }^{4}$ En diciembre 1806, el gobernador volvió a dar cuenta de la falta de comunicación oficial y hasta más de diez meses después no recibió algún que otro correo disperso y atrasado, lo cual ocurrió en octubre de $1807 .^{5}$

Justo antes de 1808, las principales autoridades en la isla se encontraron sin una dirección expresa desde la metrópoli, debiendo contar casi únicamente con las decisiones tomadas por la máxima autoridad de la isla, el capitán general Someruelos. Durante casi tres años, Cuba fue gobernada de manera casi autónoma al no recibir, apenas, órdenes directas desde Madrid. Fueron en estos años previos donde se materializaron los principales condicionantes políticos, económicos y sociales, siempre bajo la dirección política y administrativa de Someruelos al frente de Cuba. ${ }^{6} \mathrm{La}$ falta de comunicación, así como la toma de iniciativas por parte del capitán general, reforzó su autoridad en la isla y permitió que ante la llegada

3 P.C.S., "Deseos de desengaños", El Centinela de La Habana, jueves, 14 de octubre de 1813, alude a ese conflicto. Archivo Histórico Nacional de Madrid (en adelante AHN), Consejos, 21.035.

4 Someruelos a Hacienda, La Habana, 9 de enero de 1806, $\mathrm{n}^{\circ}$ 174, Archivo General de Indias (en adelante AGI), Cuba, 1753.

5 Someruelos a Hacienda, La Habana, 29 de octubre de 1807, n 194, AGI, Cuba, 1753.

- GONZÁLEZ-RIPOLL NAVARRO, M² Dolores, Cuba, la isla de los ensayos. Cultura y sociedad (1790-1815), CSIC, Madrid, 1999, p. 21. 
de la confusa información de los acontecimientos que se sucedieron en la Península, en La Habana se trataran de tomar decisiones destinadas a un mayor autogobierno.

\section{Servicio de información del Capitán General}

En segundo lugar y como una de las consecuencias directas de esta situación de autonomía de hecho, el principal modo de tratar de mantener abierta las comunicaciones con la Península y conocimiento de lo que ocurría en aquellos años tan críticos, fue mantener abierta la vía informativa con los Estados Unidos. ${ }^{7}$ El gobernador de La Habana aprovechó los testimonios de las averiguaciones que pudiesen remitirle los agentes comerciales que el gobierno tenía desplazados a los principales puertos norteamericanos. Los principales informadores fueron los cónsules destinados a Baltimore, Boston, Charleston y Nueva Orleáns. Éstos enviaban a Someruelos informes que le servían para tener una visión de la situación, en muchas ocasiones más completa que la que podía suministrar la correspondencia oficial, incluso adelantándose a los acontecimientos. Los cónsules prestaban especial atención a los asuntos comerciales, tan importantes en las relaciones de Cuba con los Estados Unidos, pero también a la política internacional en la que la isla ocupaba un lugar preferente en las intenciones expansionistas de las principales potencias de la época.

Valga como ejemplo que por los meses de marzo a mayo de 1808 llegaron a La Habana indicios de que "algo grave" estaba a punto de acontecer en España, es decir, que en Cuba se tuvieron noticias casi simultáneas, por estos adelantos, de lo que estaba pasando en la Península. ${ }^{8}$ Este tipo de informaciones permitieron en la isla prever los inminentes sucesos y prepararse ante ellos; así como una vez iniciado el conflicto bélico, los testimonios de los cónsules permitieron contrastar las noticias sobre el desarrollo de la guerra peninsular.

Por otra parte, el capitán general contó con un número importante de informadores en la propia ciudad de La Habana, que le suministraban todas aquellas noticias que pudiese ser importante para la seguridad de Cuba. De este modo recibía información que llegaba al puerto así como las que circulaban por la isla.

\section{La prensa en la isla}

En tercer lugar, el principal medio de difusión de las noticias en la época era la prensa, ${ }^{9}$ que en La Habana había surgido en 1790 con la fundación del Papel Periódico de La Habana. ${ }^{10}$ Este periódico fue difusor de los nuevos planteamientos económicos de la Sociedad Patriótica que hasta 1809 tuvieron un tratamiento preferente. Hasta 1810 hubo algunas publicaciones más como La Aurora, La Lonja Mercantil, El Mensajero y El Regañón, con una duración efímera en alguno de los casos. ${ }^{11}$ La prensa fue el foro público donde se expresaron unos ánimos cada vez más exaltados contra las autoridades peninsulares, haciéndose eco de las disputas políticas. ${ }^{12}$ El Papel Periódico siguió una línea editorial próxima a aquellos "patriotas" habaneros que ridiculizaban a los peninsulares dándoles el apelativo de godos o iberos, los cuales también hicieron uso de la prensa para su defensa. ${ }^{13}$

Sin embargo, será a partir de decreto de Libertad de Prensa de las Cortes de Cádiz de 10 de noviembre de 1810 , cuando se produzca una explosión en el número de periódicos e impresos, donde se expresaron tanto las ideas políticas y las controversias del momento, así como las noticias que se sucedían en la Península durante la guerra. Entre estos numerosos periódicos se encontraban El Lince

\footnotetext{
Someruelos a Gracia y Justicia, La Habana, 9 de enero de 1806, AGI, Cuba, 1752, n ${ }^{\circ} 132$.

Cónsul Juan Stoughton a Someruelos, Boston, 25 de marzo de 1808, 27 de abril de 1808 y 3 de mayo de 1808, AGI, Cuba, 1710.

Ver mi artículo "El incendio gaditano: libertad de prensa en La Habana", De ida y vuelta. América y España: los caminos de la cultura, Universidad de Santiago de Compostela, Santiago de Compostela, 2007, pp. 47-57.

10. Que en 1805 pasó a denominarse El Aviso y más tarde sería renombrado como El Diario de La Habana.

1 MARRERO, Leví, Cuba: Economía y sociedad, Vol. 15. Azúcar, Ilustración y conciencia (1763-1868) (VII), Ed. Playor, Madrid, 1990, pp. 21-22.

12 ZARAGOZA, Justo, Las insurrecciones en Cuba, Tomo I, Imprenta de Manuel G. Hernández, Madrid, 1872, p. 697.

13 Zaragoza, pp.168-169.
} 
(dedicado especialmente a las noticias sobre hechos de armas), El Patriota Americano, Gaceta Diaria, Mensajero Político, Económico, Literario, El Consolador, El Canario, La Mosca, El Diario Crítico, El Filósofo Verdadero, El Noticioso, El Esquife, El Correo de las Damas, Semanario Mercantil, El Robespierre español, El Censor Universal, El Hablador, La Tertulia, Ronquillo, El Reparón, La Perinola, La Cena, La Lancha, El Centinela y alguno más, ${ }^{14}$ que nos da una idea de la efervescencia que se vivió en La Habana, mientas estuvo vigente la libertad de prensa. Será en estos impresos donde quede registrada la recepción de las noticias de victorias y desastres, los movimientos políticos acontecidos en Cádiz y las nuevas propuestas, pero también los primeros movimientos insurgentes en América.

La libertad de prensa permitió que en el bando patriota arraigase "el convencimiento de que la lucha por la independencia [frente a los franceses] era tarea de todos" y sirvió para cubrir la necesidad de conocer las opiniones de instituciones y personas consideradas cualificadas. ${ }^{15}$ Por otra parte este decreto favoreció la aparición en Cuba de numerosas proclamas y escritos incendiarios, que dificultaron las labores de control por parte de las autoridades, fue considerado en su momento uno de los más funestos y trascendentales decretos de los emanados de Cádiz. Una de las indicaciones expresas de la ley era que no podía ser impedida la entrada de periódicos gaditanos, donde las expresiones radicales no eran censuradas. Tampoco podía prohibirse la circulación de las gacetas de América, por lo que llegaron periódicos de los territorios con movimientos insurgentes, donde se ridiculizaba al gobierno español y se incitaba a la secesión. El gobierno de Someruelos optó por invitar a algunos escritores para tratar de dirigir la opinión. ${ }^{16}$

\section{Rumores y comunicaciones privadas}

Por último, el puerto de La Habana era uno de los más importantes de toda América y, por su importancia económica para España, equiparable al de Cádiz. El puerto habanero era escala y destino de embarcaciones de uno y otro lado del Océano. Por sus muelles transitaban gentes de todo el mundo, los cuales transmitían todo tipo de noticias bien en las charlas con otros marineros o con los habaneros, o por la prensa que trasportaban en sus equipajes. Los chismes, rumores y confidencias compartieron espacio con los adelantos de noticias sobre hechos acaecidos, testimonio de sucesos, reseñas de enfrentamientos o noticias políticas. Es difícil conocer el alcance de estas informaciones, pero hay certera constancia de ellos.

Por otra parte, las comunicaciones privadas permitieron tener un conocimiento de la situación. Esta es una información de difícil estudio por su escasez, pero algún ejemplo nos permite hacernos una idea de cómo en las cartas personales se trasmitían noticias de interés general.

El doctor Martín de Sesé escribió desde Madrid al asesor de gobierno José de Ilincheta el 3 de marzo de 1808. Después de aconsejarle sobre temas facultativos, le dio noticias sobre el recelo que estaba causando la ocupación por parte de las tropas francesas de las plazas de Pamplona y Barcelona con

anuencia de nuestra Corte y en tono de la mayor amistad. El Rey y el príncipe de la Paz, que pueden estar en el ministerio manifestando su contento, pero los que nacimos del Ebro para allá olemos a gabachos. En fin, podemos tardar poco de salir del preñado porque Murat, Bertier y Duro, que son los tres ángeles de Bonaparte, están en Bayona y él no tardará mucho en alcanzarlos. ${ }^{17}$

14 Alguno de ellos editados a imitación de los emitidos en Cádiz

15 LA PARRA LÓPEZ, Emilio, La libertad de prensa en las Cortes de Cádiz, Biblioteca Virtual Miguel de Cervantes, Alicante, 2005. Disponible en: http://www.cervantesvirtual.com/FichaObra.html?Ref=13615\&portal=56.

16 Zaragoza, p. 246.

17 Doctor Martín de Sesé a José de Ilincheta, Madrid, 3 de marzo de 1808, Archivo Histórico Nacional de Madrid (en adelante AHN), Consejos, 21.035. 


\section{Primeros episodios de la Guerra}

Tras la puesta en conocimiento de estos condicionantes, es el momento de iniciar el estudio de la llegada de las noticias a Cuba y de la repercusión que estas tuvieron.

\section{Conjura de El Escorial y Tratado de Fontanieblau}

No hay dudas sobre que las noticias sobre la Conjura del Escorial, así como las consecuencias del Tratado de Fontanieblau, circularon por La Habana, como demuestra la celebración que comandante general de marina, Juan María de Villavicencio, hizo el 1 de enero de 1808 , al tener conocimiento de la causa de El Escorial, aunque el capitán general consideró que no había motivo de celebración. ${ }^{18}$ El tema debía ser de dominio público cuando un anónimo emitido en La Habana, en julio de 1808 recordaba el caso y lo esgrimía para ejemplarizar como un ejemplo de justicia y valor:

El mismo Consejo de Castilla acaba de darnos un testimonio de integridad que asombra. Véase la causa seguida desde octubre contra nuestro rey Fernando, entonces príncipe de Asturias y contra el duque del Infantado y canónigo Ezcoiquiz y otros individuos a quienes se atribuía alta traición. Véase la acusación fiscal declarándolos reos y véase el poderoso influjo que movía la machina. Pues a pesar de todo esto absolvió el consejo a los supuestos reos por consulta de 25 de enero de este mismo año. ${ }^{19}$

Desde Boston, Someruelos tuvo en mayo el adelanto de noticias sobre graves sucesos, según la información fechada el 18 de febrero en Londres, que aseguraba "no tardaremos mucho en saber acontecimientos pasmosos" ${ }^{20}$ Posiblemente alguna preocupante noticia más debió llegar al puerto en las numerosas embarcaciones que arribaban, pues para el mes de mayo habían pasado más de 2 meses desde los acontecimientos de Aranjuez.

\section{Motín de Aranjuez y 2 de mayo}

A fines de abril, el cónsul en Boston dio nuevas noticias sobre la llegada de una información fechada el 25 de marzo en Londres, que se hacía eco de rumores de grandes alteraciones que iban a sacudir la Península. ${ }^{21}$ Con toda seguridad estos debían estar referidos al motín de Aranjuez.

Dada la dificultad en las comunicaciones, hasta el 9 de junio de 1808 no fueron recibidas de manera oficial las notificaciones de las consecuencias del motín. ${ }^{22}$ El día 10 Someruelos, acusó recibo de la real orden de 18 de marzo sobre la destitución del príncipe de la Paz como Generalísimo y Almirante, ${ }^{23}$ así como la de 19 de marzo sobre la abdicación de Carlos IV en el Príncipe de Asturias. ${ }^{24}$

No hay dudas sobre que el conocimiento de los sucesos de Aranjuez en La Habana debió ser anterior a estas fechas, pues habían pasado casi tres meses de los acontecimientos y, a pesar de las dificultades, algún barco debió haber llegado al puerto mucho antes con las nuevas. Y no era esta una noticia que pudiera pasar desapercibida. Por ejemplo el 31 de mayo esta misma noticia había sido recibida en Mérida de Yucatán, procedente de Cádiz, tras cuarenta y un días de navegación. ${ }^{25}$

Alguna información complementaria debía tener Someruelos cuando el mismo mes de junio dio al gobernador de Santiago de Cuba la disposición de no entregar el mando si no portaba la posible

\footnotetext{
P.C.S., "Deseos de desengaños", El Centinela de La Habana, jueves, 14 de octubre de 1813, AHN, Consejos, 21.035.

J.M.P.M, La Habana, 20 de julio de 1808, AHN, Estado, 59, L.

Stoughton a Someruelos, Boston, 25 de marzo de 1808, AGI, Cuba, 1710. Esta comunicación fue respondida el 2 de mayo de 1808 por Someruelos.

21 Stoughton a Someruelos, Boston, 27 de abril de 1808, AGI, Cuba, 1710. La noticia fue contestada por Someruelos el 8 de junio.

22 Someruelos a Guerra, La Habana, 10 de junio de 1808, $n^{\circ} 2364$, AGI, Cuba, 1746.

23 Someruelos a Hacienca, La Habana, 10 de junio de $1808, n^{\circ} 213$, AGI, Cuba, 1753.

24 Someruelos a Guerra, La Habana, 10 de junio de $1808, n^{\circ} 2363$, AGI, Cuba, 1746.

25 Benito Pérez, Capitán General de Yucatán a Estado, Mérida, 23 de Junio de 1808, n 31, AGI, Estado, 57.
} 
orden al efecto el "cúmplase" firmado por él mismo. ${ }^{26} \mathrm{El}$ temor al peligro de un intento de usurpar el poder no podía hacer referencia a otra cuestión que los sucesos del 2 de mayo y más específicamente a las abdicaciones de Bayona.

Fue en la noche del 14 de julio cuando entró en el puerto habanero un barco que trasportaba al intendente electo Juan de Aguilar. Los rumores, que muy posiblemente estaban circulando por La Habana desde hacía días, tomaron carta de naturaleza. Aguilar dio cuenta de manera oficial de que toda la familia real española había sido internada en Francia, que había habido "una gran revolución a la salida de Madrid de las últimas personas reales, con mucha efusión de sangre, y que sólo quedaban sin guarnición francesa, a su salida, las plazas de Cádiz y Ceuta", así como del nuevo cambio de dinastía pretendido por Napoleón. ${ }^{27}$

El 15 de julio se reunió el Cabildo se trataron las gravísimas noticias que portaba Aguilar y la decisión que tomó fue que debía precaverse que de un momento a otro llegase un navío despachado por Bonaparte exigiendo su reconocimiento en la isla. ${ }^{28}$

\section{Proclamación de Fernando VII y declaración de guerra a los franceses}

Durante la toma de posesión del nuevo intendente Aguilar debió tener lugar una reunión de los ministros y oficiales de hacienda, ${ }^{29}$ donde seguro se trató la situación en la Península, haciendo ver que allí era mayoritaria la adhesión a Fernando VII.

Para el 18 de julio de 1808 Someruelos, por las noticias comunicadas por Aguilar, tenía constancia oficial de los sucesos del 2 de mayo en Madrid y es más que posible que hubiese sido informado del caso de la revolución "santa" de Sevilla, en que la indecisión de las autoridades motivó la insurrección general del pueblo en la noche del 26 de mayo de $1808 .{ }^{30}$ Es difícil de creer que no hubiesen llegado las noticias de Cádiz, en que una turba había acabado con la vida del capitán general de Andalucía, marqués del Socorro. ${ }^{31}$ Con estos ejemplos, Someruelos debió tomar conciencia de que debía adoptar decisiones para evitar una situación similar. Para entonces se había logrado un consenso en el cabildo habanero, en sesión convocada por el gobernador, para proceder la proclamación de Fernando VII, ${ }^{32}$ que se llevó a cabo el 20 de julio. ${ }^{33} \mathrm{El} 22$ de julio el gobernador dio cuenta al Cabildo de que mandaba imprimir lo más brevemente posible la declaración de guerra a Francia y el armisticio con Gran Bretaña, publicados por la Junta de Sevilla. ${ }^{34}$ A pesar de la actitud dubitativa de las autoridades, la población en general mostró su exaltación al conocer las noticias desde el primer momento. ${ }^{35}$

En el resto de la isla se tardó algún tiempo en proclamar al nuevo monarca, dadas las distancias que debían recorrer las comunicaciones. En Bayamo se produjo el 5 de agosto; en Baracoa tuvo lugar el

\footnotetext{
26 Sebastián Kindelán, gobernador de Santiago de Cuba, a Someruelos, Santiago de Cuba, 30 de junio de 1808, $\mathrm{n}^{\circ} 70$, Archivo Nacional de Cuba (en adelante ANC), Asuntos políticos, 142.

27 ARMAS MEDINA, Fernando de, "La Audiencia de Puerto Príncipe (1775-1853)", Anuario de Estudios Americanos, Vol. XV, Art. 5, Escuela de Estudios Hispano-Americanos, CSIC, Sevilla, 1958, pp. 273-370.

28 Esta noticia no aparece relatada en las actas, hasta que el 23 de septiembre el capitán general presentó oficio con la queja por "el olvido", por tanto lo que conocemos de lo ocurrido en dicha reunión es una elaboración hecha a posteriori. Anexo al acta del cabildo ordinario, 20 de septiembre de 1808, Archivo de la Oficina del Historiador de la ciudad de La Habana (en adelante AOHCH), Actas de Cabildo, 1808-1809, fol. 114.

29 Intendente Juan de Aguilar a Someruelos, 17 de julio de 1808, AGI, Cuba, 1595.

30 MORENO ALONSO, Manuel, La Revolución "Santa" de Sevilla, Caja San Fernando de Sevilla y Jerez, Sevilla, 1997, p. 25.

31 SOLÍS, Ramón, El Cádiz de las Cortes. La vida en la ciudad en los años 1810 a 1813, Silex, Madrid, 1987, p. 147; Pettenghi, José, "San Martín en Cádiz. Camino de América", en Vida española del General San Martín, de Lago Carballo, Antonio (coord.), Instituto Español Sanmartiniano, Madrid, 1994, pp. 181-186; Pacífico Otero, José, Historia del libertador don José de San Martín, Vol. 1, Buenos Aires, 1978, pp. 114-120. Las relaciones con San Martín se deben a que el futuro Libertador, formaba parte del cuerpo de guardia del marqués del Socorro.

32 Cabildo ordinario, 18 de julio de 1808, AOHCH, Actas de Cabildo, 1808-1809, fol. 87.

33 Certificación del escribano Miguel Méndez, 20 de julio de 1808, AOHCH, Actas de Cabildo, 1808-1809, fol. 89-91.

34 Cabildo ordinario, 22 de julio de 1808, AOHCH, Actas de Cabildo, 1808-1809, fol. 87-91.

35 Cabildo ordinario, 18 de julio de 1808, AOHCH, Actas de Cabildo, 1808-1809, fol. 87.
} 
14 de agosto, y el 20 del mismo mes en Holguín. ${ }^{36}$ En Santiago de Cuba ésta no tuvo lugar hasta el 13 de septiembre de $1808 .{ }^{37}$

\section{La Junta de Sevilla y la Junta Central}

Una vez conocida la situación en la Península, los cubanos trataron de adaptarse a la nueva coyuntura política, y si bien en La Habana hubo un primer intento autonómico, este no fraguó y la isla se decantó por seguir las propuestas políticas que se fueron configurando por las autoridades patrióticas.

\section{Propuesta juntista}

El 17 de julio había llegado un buque portando documentos de varias Juntas de la Península que habían decidido resistir a los franceses y se "declaraban tan soberanas como la de Sevilla" ${ }^{38}$ El mismo día los principales de la capital presentaron al capitán general el plan para establecer en La Habana una Junta Superior de gobierno "revestida de igual autoridad a las demás de la península de España".

La elaboración de un proyecto de este tipo fue posible por el conocimiento previo que tenían de la situación peninsular los promotores del plan, los cuales solían reunirse en tertulias ligadas con los postulados ilustrados, donde seguro se debían tratar las noticias y rumores.$^{39}$ Fue tras la proclamación de Fernando VII cuando se pudo plantear el proyecto juntista, que explícitamente apoyó el gobernador con su oficio de 22 de julio en el que reconocía "el independiente derecho que tienen las distintas provincias de gobernarse por sí mismas". ${ }^{40}$ En el cabildo celebrado ese mismo día se acordó que se examinasen los avisos y papeles con noticias de los sucesos acontecidos en España y de lo que se había ejecutado en las demás provincias. ${ }^{41}$

El 26 de julio se presentó oficialmente la proposición de establecer "una Junta Superior de Gobierno, que [...] cuide y provea todo lo conducente a nuestra existencia política y civil, bajo el suave dominio de nuestro adorado monarca", propuesta que fue firmada por 73 miembros de la sociedad habanera. ${ }^{42}$ Sin embargo, era un número escaso, más aún cuando se había estimado que debían ser unos 200 los firmantes. ${ }^{43} \mathrm{Al}$ día siguiente comenzó a hacerse patente el rechazo al plan especialmente entre el público en general que había sido informado por medio de pasquines que calificaban a la Junta como "tiránica e independiente". La inestabilidad política y la falta de apoyos significativos hicieron a sus inductores retirar proyecto finalmente, tras lo cual cesó la inquietud de la población. ${ }^{44}$

\section{Excitación patriótica}

Tras los acontecimientos de julio en La Habana, Someruelos trató de normalizar la situación, pues la llegada numerosas noticias, con informaciones de todo tipo, muchas de ellas sin contrastar, estaban exaltando los ánimos en la isla. Por ejemplo, en Santiago de Cuba el 21 de agosto habían llegado informes desde Jamaica que aseguraban que "José Bonaparte había sido derrotado y muerto con 19.000 hombres que pretendieron forzar el paso [...] de Cataluña", que 12,000 franceses habían sido rodeados en el castillo de Monfui y que cuatro generales españoles habían sido degollados por estar relacionados con Napoleón; o que se estaba organizando en Galicia un ejército de 29.000 hombres que debía avanzar sobre Madrid. ${ }^{45}$

\footnotetext{
Actas de Cabildo de Bayamo, Baracoa y Holguín, Santiago de Cuba, 24 de septiembre de 1808, $\mathrm{n}^{\circ}$ 57, ANC, Asuntos Políticos, 209. Testimonio del Acta de Cabildo, Santiago de Cuba, 13 de septiembre de 1808, $\mathrm{n}^{\circ}$ 113, ANC, Asuntos Políticos, 142.

Zaragoza, p. 182.

KUETHE, Allan J., Cuba, 1753-1815. Crown, Military and Society, The University of Tennessee, Knoxville, 1986, p. 163. Someruelos al Ayuntamiento, La Habana, 22 de julio de 1808, AGI, Cuba, 1627.

Cabildo ordinario, 22 de julio de 1808, AOHCH, Actas de Cabildo, 1808-1809, fol. 87-91.

MORALES Y MORALES, V., Iniciadores y primeros mártires de la revolución cubana, vol.1, La Habana, 1931, pp. 22-23.

GUERRA Y SÁNCHEZ, Ramiro, Historia de la nación cubana, Tomo III, La Habana, 1952, pp. 18-20.

Documentación sobre el juicio de residencia de Someruelos, AHN, Consejos. 21.035.

Diario muy reservado de la Secretaría de gobierno, Santiago de Cuba, del 18 de julio de 1808 al 8 de marzo de 1809, AGI, Cuba, 1782-B.
} 
La noticia de la victoria del general Castaños ante las tropas napoleónicas de Dupont en Bailén alentó el patriotismo en la isla de Cuba. El secretario del Consejo de Indias había dado cuenta a Someruelos en cartas de 12 de agosto y 18 de septiembre "sobre los triunfos gloriosos que entre ambas épocas han continuado consiguiendo nuestros ejércitos en la Península" ${ }^{46}$ Las noticias fueron hechas circular con profusión por toda la isla con el fin de elevar la moral y para alentar el patriotismo en Cuba. Coincidiendo con las noticias oficiales, en diciembre 1808 se observó que faltaban soldados de la dotación de La Habana, por los deseos de un gran número de estos de presentarse en los ejércitos expañoles de la Península para combatir al francés. ${ }^{47}$

En La Aurora Extraordinaria del 8 de diciembre de 1808, Someruelos había hecho publicar las disertaciones ocurridas en el Senado de Francia por las derrotas del verano anterior. ${ }^{48} \mathrm{La}$ intención era demostrar la importancia del suceso cuando en el propio París se discutía sobre él. Las victorias en la Península habían servido a Someruelos para haber "excitado a los habitantes de estos reinos, como lo han verificado haciendo grandes esfuerzos". ${ }^{49}$

Casi desde la misma llegada de las primeras noticias de julio, la población cubana reaccionó con la intención de colaborar en la lucha que se iniciaba en la Península. En reunión extraordinaria del Cabildo el 24 de julio se decidieron las aportaciones económicas para sustentar a los soldados que se enfrentaban a los franceses, ${ }^{50}$ y el 8 de agosto de 1808 Someruelos dirigió una proclama a los habitantes de la isla para que colaborasen con dinero para apoyar la causa a favor de Fernando VII. ${ }^{51}$

La primera remesa enviada desde La Habana en noviembre ascendió a 93.178 pesos en barras de oro y plata, dinero, alhajas y frutos (principalmente azúcar y tabaco), donativo voluntario de los habaneros, a los que se sumaron 14.755 pesos enviados por catalanes que habitaban en Cuba. ${ }^{52} \mathrm{El} 19$ de mayo de 1809 se leyó ante el cabildo habanero el agradecimiento de la Suprema Junta Central, por los donativos patrióticos enviados desde La Habana. ${ }^{53}$

Pero la exaltación tuvo una vertiente de exacerbación xenófoba contra los franceses en la isla, donde constituían una colonia muy importante en número e importancia económica y social. El trato moderado que las autoridades dispensaron a los galos residentes en la isla fue muy mal entendido por el público en general. ${ }^{54}$ En Santiago de Cuba, donde los franceses suponían el $22 \%$ de la población, ${ }^{55}$ pronto se reprodujeron los ataques a los mismos por medio de pasquines. ${ }^{56}$ Las noticias llegadas en enero de 1809 que daban cuenta de la nueva capitulación de Madrid, elevaron el tono de los ataques a los franceses hasta que en marzo acabó por desencadenar un tumulto contra ellos en La Habana, que hizo ver a las autoridades que debían ser expulsados de la isla. ${ }^{57}$

46 Someruelos al secretario de Consejo de Indias, La Habana, 12 de diciembre de 1808, AGI, Cuba, 1754.

47 Someruelos a Guerra, La Habana, 30 de enero de 1809, n²403, AGI, Cuba, 1747.

48 La Aurora Extraordinaria del jueves 8 de diciembre de 1808, número 550. Someruelos a Guerra, La Habana, 14 de diciembre de 1808, $\mathrm{n}^{\circ} 2385$, AGI, Cuba, 1746.

49 Someruelos al Secretario del Consejo de Indias, La Habana, 12 de diciembre de 1808, AGI, Cuba, 1754.

so Cabildo extraordinario, 24 de julio de 1808, AOHCH, Actas de Cabildo, 1808-1809, fol. 91.

s. La Habana, 7 de agosto de 1808, AGI, Cuba, 1769.

:: Someruelos a Hacienda, La Habana, 28 de diciembre de 1808, n 218, AGI, Cuba, 1753. Lucena Salmoral, Manuel, "La Junta Central Suprema de España e Indias y el comercio americano", Estudios de Historia social y económica de América, Separata n ${ }^{\circ}$, Universidad de Alcalá de Henares, 1985 , p. 63.

Cabildo ordinario, 19 de mayo de 1809, AOHCH, Actas de Cabildo, 1808-1809, fol. 346-356.

* Sobre el trato dado a los franceses en la sociedad habanera y las autoridades ver mi trabajo "La élite habanera y los refugiados tameses de Saint Domingue". Élites Urbanas en Hispanoamérica, Secretariado de Publicaciones de la Universidad de Sevilla, Sevile 3005 .

= E- Ṕ́REZ DE LA RIBA, Juan, El Barracón y otros ensayos, Ed. Ciencias Sociales, La Habana, 1975, p. 370.

IT.- 


\section{Establecimiento de la Junta Central}

Debido a la demora en las comunicaciones oficiales, cuando el cabildo habanero finalmente reconoció la autoridad de la Junta de Sevilla, esta ya había cesado sus funciones. ${ }^{58}$ La confusión produjo que el día 22 octubre se considerase que de manera indudable debía prestarse obediencia a la nueva Junta Central que creían constituida en 18 de septiembre en La Mancha. ${ }^{59}$ Aunque la noticia era falsa sirvió para que las autoridades cubanas estuviesen sobre aviso del nuevo cambio político.

Para el 8 de noviembre llegó la noticia, ya confirmada, de la instalación de la Junta Central, por la llegada al puerto de Matanzas de un buque procedente de Sanlúcar de Barrameda, información que el gobernador había hecho publicar en La Aurora. El 25 de noviembre por fin se recibió en La Habana la notificación oficial de la instauración de la Junta Central Gubernativa, que había tenido lugar en Aranjuez justo 2 meses antes. El hecho tras difundirse al público fue celebrado con los mismos festejos que tuvieron lugar tras la designación de Godoy como Almirante, con salvas, iluminación, repique general de campanas, funciones de teatro, danzas y fuegos artificiales. ${ }^{60}$

El 17 de mayo de 1809 fue recibida en La Habana la real orden de 22 de enero del mismo año, relativa a que los reinos, provincias e islas que formaban los dominios de España e Indias debían tener representación nacional inmediata para formar parte de la Junta Central. En Cuba la elección de un diputado por cada una de las capitales de cabeza de partido que constituían los ayuntamientos de $\mathrm{La}$ Habana y Santiago de Cuba ${ }^{61}$ Se suscitó entonces la duda de si sólo debían considerarse como capitales de partido las dos dichas ciudades, pues la villa de Puerto Príncipe pretendió acceder a tal distinción, por ser cabeza de distrito y corte de justicia. ${ }^{62}$ Someruelos intervino y consideró que sólo las dos ciudades con gobernador en la isla debían tener representación. ${ }^{63}$ Pero en septiembre de 1809 el tribunal de la Real Audiencia apreció que debían ser admitidas las ciudades de Trinidad y Puerto Príncipe. El capitán general decidió esperar a un fallo de la Junta Central sobre qué debía hacerse. ${ }^{64}$ Esta solución motivó que el proceso quedase paralizado con el perjuicio indudable de que la isla de Cuba no enviase sus representantes a la Junta.

\section{Cuestiones económicas}

El 9 de mayo de 1809 se propuso la creación de un nuevo plan de comercio con arreglo de los aranceles, pues la crisis económica que sufría la isla había ido creando una opinión favorable de aranceles más liberales que estimularan las importaciones. ${ }^{65}$ Sin embargo, las autoridades de la Junta Central se opusieron a esta solución pues trataba de mantener las prácticas monopolísticas a toda costa, para asegurarse unos ingresos que eran muy necesarios. ${ }^{66}$ Someruelos trató de eludir estas prohibiciones pero el 23 de septiembre de 1809 llegó a La Habana la real orden de 10 de julio del mismo año que obligaba a cumplir de manera estricta las órdenes contra el comercio con extranjeros ${ }^{67}$ suspendiendo el plan de 9 de mayo. ${ }^{68}$ La persistencia en el mantenimiento de las medidas contrarias a los intereses cubanos, colmó la paciencia de los productores de la isla, lo cuales elevaron una protesta por la situación:

Si estas (prácticas de comercio libre) se permiten en Cádiz, por ejemplo, Cádiz prosperará. Si se niega a los demás puertos, serán miserables sin remedio. La importación o

\footnotetext{
Zaragoza, pp. 190-192; Cabildo ordinario, 2 de marzo de 1809, AOHCH, Actas de Cabildo, 1808-1809, fol. 169-172. GUERRA Y SÁNCHEZ, Ramiro, op. cit. p. 25.

Cabildo ordinario, 22 de octubre de 1808, AOHCH, Actas de Cabildo, 1808-1809, fol. 125-129.

Someruelos al secretario de Consejo de Indias, La Habana, 7 de diciembre de 1808, AGI, Cuba, 1754; Zaragoza, p. 184.

Someruelos a Hacienda, La Habana, 9 de junio de 1809, $\mathrm{n}^{\circ} 225$, AGI, Cuba, 1753.

Cabildo ordinario, 28 de julio de 1809, AOHCH, Actas de Cabildo, 1808-1809, fol. $397-399$.

Someruelos a Hacienda, La Habana, 29 de agosto de 1809, $\mathrm{n}^{\circ}$ 241, AGI, Cuba, 1753.

Cabildo ordinario, 1 de septiembre de 1809, AOHCH, Actas de Cabildo, 1808-1809, fol. 414-420.

Someruelos a Hacienda, La Habana, 12 de mayo de 1809, $\mathrm{n}^{\circ} 224$ AGI, Cuba, 1753.

Someruelos a Hacienda, La Habana, 20 de junio de 1809, $\mathrm{n}^{\circ} 230$. AGI, Cuba, 1753.

${ }_{67}$ Ibidem. La Habana, 27 de septiembre de $1809, \mathrm{n}^{\circ} 243$.
} 
exportación con la nación solamente, proveerá a algunos lugares, no a todos. Surtirá de unos artículos, no de otros y a precios subidos sin proporción. Hará feliz a un puñado de hombres e infeliz al resto y la nación aislada o replegada, digámoslo así, dentro de sí misma, decaerá al fin por falta de expansión, de luces y de recursos. [...]Merecemos más consideración que la gran masa del Estado, pues somos los que constituyen su riqueza. ¿Por qué se concede a los mismos españoles que están bajo la dominación enemiga un tráfico que a nosotros, hallándonos libres en nuestro país, se nos niega con nuestros reinos? ${ }^{69}$

Se hace evidente que en La Habana se estaba al tanto de todo lo que estaba aconteciendo en la Península y querían trato al menos igual que cualquier otro español del otro lado del Océano.

\section{Consejo de Regencia y Cortes}

De nuevo Cuba de adaptó al nuevo cambio político, que en este caso iba a significar mayores dificultades para la isla.

\section{Elección a Cortes}

El 10 de enero de 1810 llegó a La Habana la real orden de 6 de octubre con las reglas que debían observarse en las elecciones a diputados a Las Cortes en América. El Cabildo respiró aliviado pues las reglas parecían claras y pronto podrían tener su representante en el cuerpo nacional. ${ }^{70} \mathrm{Sin}$ embargo, hasta el 26 de abril de 1810 no se procedió a la proclamación del Supremo Consejo de Regencia, a cuya instalación siguió la convocatoria de Cortes Generales, lo que dio lugar a la expresión de exaltación patriótica por parte del Cabildo. ${ }^{71}$

En la elección realizada en La Habana, la suerte había recaído en el teniente regidor alguacil del ayuntamiento Andrés Álvarez de Jáuregui. ${ }^{72}$ Santiago de Cuba eligió como diputado para las Cortes extraordinarias al licenciado Tomás del Monte y Mesa, aunque este renunció al cargo. La Audiencia determinó que el diputado representante de dicha ciudad fuese el provisor y vicario general de La Habana Juan Bernardo O'Gaban, que fue el tercer sujeto nombrado. ${ }^{73}$ Después de las dificultades y resistencias opuestas desde la elección del representante a la Junta Central, la oligarquía habanera logró hacer imponer su criterio y dominar la delegación que debía defender los intereses cubanos en La Cortes. El 25 de noviembre de 1810, tras un Tedeum en la catedral de La Habana, se hizo el juramento a las Cortes, en público por considerarlo el capitán general "muy conveniente y conforme" al orden de lo que se había hecho en Cádiz. ${ }^{74}$ El 12 de enero de 1811 partió a Cádiz Andrés Álvarez de Jáuregui, diputado electo de La Habana para las Cortes Generales. ${ }^{75}$ Sin embargo, la dilación en las discusiones y demoras en las elecciones hicieron que cuando Jáuregui llegó a la Península algunas decisiones importantes para Cuba hubiesen sido tomadas, a pesar de lo cual se mostró como el más participativo de los representantes cubanos en las sesiones.

Las deliberaciones en las Cortes fueron seguidas en Cuba con gran atención como queda representado en el siguiente aviso:

Viendo la utilidad que resulta de la lectura del Diario de las Cortes generales y extraordinarias de la nación; el ansia con que lo han solicitado algunos curiosos en el correo y el empeño de otros a suscribirse para él o a lo menos que se les tenga presente para cuando lleguen otras colecciones del

68 El peligro de ser acusado de deslealtad fue determinante a la hora de tomar esta decisión.

69 Grupo de vecinos (138) de La Habana al Ayuntamiento, La Habana, 10 de septiembre de 1809, anexo al Cabildo ordinario, 20 de octubre de 1809, fol. 450-455, AOHCH, Actas de Cabildo, 1808-1809.

7o Cabildo ordinario, 19 de enero de 1810, AOHCH, Actas de Cabildo, 1 de enero de $1810 / 6$ de julio de 1810, fol. $34-43$.

71 Cabildo extraordinario, 26 de abril de 1810, AOHCH, Actas de Cabildo, 1 de enero de 1810/6 de julio de 1810, fol. $191-199$.

72 Someruelos a Gracia y Justicia, La Habana, 7 de septiembre de 1810, $\mathrm{n}^{\circ}$ 241, AGI, Cuba, 1752.

73 Ibidem, La Habana, 30 de septiembre de $1811, \mathrm{n}^{\circ} 313$.

74 Ibidem, La Habana, 29 de noviembre de $1810, \mathrm{n}^{\circ} 251$.

75 Ibidem, La Habana, 12 de enero de $1811, \mathrm{n}^{\circ} 258$. 
mismo diario, se hace saber que la administración de correos admitirá suscriptores de los que los quieran. ${ }^{76}$

\section{Reivindicaciones cubanas}

Con la constitución del primer Consejo de Regencia de España ya en Cádiz en enero de 1810, el consulado de la capital andaluza consideró que era el momento de recuperar los derechos de su antiguo monopolio, pues el nuevo gobierno constituido puso el manejo de la Hacienda española en manos de una junta nombrada por el ayuntamiento gaditano, en agradecimiento a sus innumerables esfuerzos económicos hechos. ${ }^{77}$ Fue la peor noticia recibida en La Habana entre aquellos que aspiraban a una mayor libertad en el comercio. ${ }^{78}$ El cabildo habanero expresó su malestar exigiendo que cesase "ya el egoísmo que quieren cubrir con la expresión de comercio nacional". ${ }^{79}$ Las noticias que diariamente llegaban a La Habana por vía oficial o privada daban la impresión de que Cádiz se obviaban las preocupaciones de los americanos, sobre todo en lo referente a cuestiones económicas. ${ }^{80}$

Consulado y Ayuntamiento elevaron el tono en sus representaciones al gobernador, con la intención de recuperar el reglamento de 9 de mayo de 1809. Someruelos era consciente de que de no permitir alguna medida liberalizadora la cuestión podía ser resuelta por la abierta desobediencia, por lo que volvió a permitir el comercio con neutrales. ${ }^{81}$ En Cuba se conocía la entrada de los franceses en Andalucía, por lo que eran conscientes de que esta situación vendría a dificultar más aún las relaciones comerciales con la Península. Además, hay que tener presente que de manera simultánea se estaban produciendo en la isla las primeras pretensiones secesionistas, como ocurrió el 19 de octubre de 1809 cuando fue arrestado en La Habana Román de la Luz, ${ }^{82}$ o comenzaron a aparecer en Puerto Príncipe pasquines injuriosos contra las autoridades en el mismo mes. ${ }^{83}$

\section{Malestar en Cuba}

El 21 de enero de 1811 la llegada de un periódico gaditano hizo pública la declaración de libertad de prensa promulgada por las Cortes el 10 de noviembre de $1810 .{ }^{84} \mathrm{Como}$ hemos visto antes, esto supuso una difusión nunca antes vista de la opinión publicada en La Habana. La situación de guerra motivó que se publicasen expresiones contra la tiranía y el mal gobierno, en las ciudades cubanas se hablaba abiertamente de la lucha por la libertad. ${ }^{85}$ La prensa sirvió para difundir ideas dentro del concepto general de "libertad", que no estaba contemplada por la mayor parte de las autoridades o la élite cubana. El asunto que levantó más resquemores fue se publicasen opiniones contra la esclavitud y a favor de la abolición.

Con la propuesta el 2 de abril de 1811 por el diputado asturiano Agustín Argüelles para abolir la trata de esclavos, ${ }^{86}$ se llegó al punto culminante de desencuentro entre los cubanos y las Cortes. Andrés de

\footnotetext{
76 Aviso, Diario de La Habana, $\mathrm{n}^{\circ} 216$, jueves, 4 de abril de 1811, Biblioteca Nacional de España (en adelante BNE), Sala de Prensa, REV micro/ 2011.

77 El total de los préstamos efectuados a la Junta Central ascendieron entre abril y noviembre de 1809 a 2.219 .25455 pesos. En Lucena Salmoral, Manuel, "Los préstamos del Consulado de Cádiz a la Junta Central Suprema", Anales de la Universidad de Murcia, Vol. XXXII-N 1 1-2-3-4, Filosofía y Letras, Curso 1973-74, Ed. 1977, p. 15

78 GUERRA Y SÁNCHEZ, p. 28-29. El todo poderoso lobby gaditano usó todos los medios a su disposición para rechazar las exigencias hechas desde América; CUENCA TORIBIO, José Manuel, La Guerra de la Independencia: un conflicto decisivo (18081814), Encuentro, Madrid, 2006, p. 229

79 Cabildo extraordinario, 28 de marzo de 1810, AOHCH. Actas de Cabildo, 1 de enero de 1810/ 6 de julio de 1810, fol. 145-172.

80 Zaragoza, pp. 244-245.

81 Someruelos a Hacienda, La Habana, 22 de junio de 1810, $n^{\circ} 298$, AGI, Cuba, 1753.

82 Román de la Luz fue puesto en libertad y vuelto a detener en 4 de octubre de 1810 , cuando se hallaba en la preparación de una conjura de mayores proporciones. Franco, José Luciano, Las conspiraciones de 1810 y 1812, La Habana, 1977, p. 45-48.

83 Someruelos a Gracia y Justicia, La Habana, 30 de noviembre de 1809, $\mathrm{n}^{\circ}$ 197, AGI, Cuba, 1752; SEVILLA SOLER, María del Rosario, "Cuba: Los primeros enfrentamientos políticos (1808-1826)", Arbor CXLIV, 567, marzo, 1993, p. 85.

84 MARRERO, Leví, Cuba: economia y sociedad, Vol, 9, Ed. Playor, Madrid, 1983, p. 23.

s.5 Zaragoza, p. 193-194.

86 MARTÍNEZ CARRERAS, José U., "La abolición de la esclavitud en España", en Solano, Francisco de, y Agustín Gimerá Ravina, Esclavitud y Derechos Humanos. La lucha por la libertad del negro en el siglo XIX, CSIC, Madrid, 1990, p. 67-68.
} 
Jáuregui, como diputado a Cortes manifestó que esa proposición significaba comprometer la tranquilidad de la isla ${ }^{87} \mathrm{Al}$ llegar la noticia a La Habana se produjo un estado de consternación especialmente entre los hacendados. Ayuntamiento, Consulado y Sociedad Patriótica reclamaron la suspensión la propuesta, ${ }^{88}$ y protestaron por

la intempestiva moción que se hizo en las Cortes para abolir el tráfico de negros, publicada allí con todos los horrores de la esclavitud y transcendidas aquí de un modo inexacto y placentero que puede excitar en algunos de nuestros esclavos, comúnmente bien tratados, falsas ideas de su libertad. ${ }^{89}$

El gobernador Someruelos dio cuenta a las Cortes de la necesidad de acabar con las discusiones sobre la esclavitud, pues el peligro era presente y estaba en juego la propia isla. ${ }^{90}$

Es muy grande, señor; la sensación que ha hecho en estos habitantes y muy tristes las especies que se susurran en esta capital y que irán cundiendo por los campos y por todas las demás poblaciones de la isla que excita toda la vigilancia del gobierno. ${ }^{91}$

La presión realizada desde Cuba, entendidas en tono muy amenazador, sirvió para que las Cortes archivaran el debate abolicionista. ${ }^{92}$ Cuando en Cádiz se comprobó que la resistencia cubana a la abolición era muy seria, los diputados aceptaron que no hubiese modificación en esta cuestión..$^{93} \mathrm{La}$ demostración de que parecía real el temor cubano fue la revuelta de esclavos que entre marzo y abril de 1812 tuvo en las inmediaciones de La Habana. ${ }^{94}$ Seguidamente, Cuba logró una regulación del comercio muy favorable entre los meses de septiembre y octubre de 1811, que colmaba las aspiraciones que venían manifestando comerciantes y productores habaneros.

La toma de estas decisiones coincidió con la mayor supremacía francesa en la Península, el momento más difícil para la Regencia, recluida casi exclusivamente en Cádiz, y que cifraba sus esperanzas en el apoyo de las posesiones americanas y sus aliados británicos. ${ }^{95}$

\section{La Constitución}

El 13 de junio de 1812 llegó a La Habana, la Constitución política de la monarquía española, la cual fue jurada solemnemente dos días después, presidiendo el acto el nuevo capitán general Juan Ruiz de Apodaca, que había tomado posesión 3 meses antes, sustituyendo a Someruelos, que aún permanecía en la isla. ${ }^{96}$

87 MORENO FRAGINALS, Manuel, El Ingenio, Ed. Crítica, Barcelona, 2001, p. 111.

88 Representación del Ayuntamiento, Consulado y Sociedad Patriótica de La Habana, 20 de julio de 1811. En CHUST, Manuel, La cuestión nacional americana en las Cortes de Cádiz (1810-1814), Centro Francisco Tomás y Valiente, Valencia, 1999, p.110.

99 Ayuntamiento de La Habana a Someruelos, AGI, Santo Domingo, 1286. En TORNERO TINAJERO, Pablo, Crecimiento económico y transformaciones sociales. Esclavos, hacendados y comerciantes en la Cuba colonial (1760-1840), Ministerio de Trabajo y Seguridad Social, Madrid, 1996, p. 82.

90 MORENO FRAGINALS, Cuba/España, España/Cuba. Una historia común, Grijalbo Mondadori, Barcelona, 1995, p. 190.

91 Someruelos a Las Cortes, La Habana, 27 de mayo de 1811, AHN, Sección Nobleza (Toledo) (en adelante SN), Almodóvar, C.36, D.1.

${ }_{92}$ En Rieu-Millan, p. 169. Un ejemplo fue la ratificación de la liberación por el presidente e la Audiencia de Guatemala de 506 esclavos de Omoa por haber ofrecido 1.280 pesos al gobierno español, tras la intervención del diputado por Guatemala Antonio Larrazábal en enero de 1812.

93 PÉREZ LEDESMA, Manuel, "Las Cortes de Cádiz y la sociedad española", en Artola. Miguel (ed.), Las Cortes de Cádiz, Marcial Pons, Madrid, 1991, p. 184.

94 Someruelos a Gracia y Justicia, La Habana, 12 de abril de 1812, $\mathrm{n}^{\circ} 350$, AGI, Cuba, 1752. Ver en Franco, Las conspiraciones de 1810 y 1812, La Habana, 1977, pp. 213-220.

95 SUPERVIELLE HERNÁNDEZ, Manuel Guillermo, La burguesía gaditana en la crisis del Antiguo Régimen, Centro de Estudios Constitucionales 1812, Universidad de Cádiz, 2007, pp. 177-182.

96 Zaragoza, p. 258. 
El tiempo entre la instalación de los ayuntamientos constitucionales y las diputaciones provinciales y su supresión el 4 de mayo de 1814 no fue suficiente para poder desarrollar sus utilidades y sólo sirvió este periodo para permitir una lucha política expresada a través de los numerosos periódicos. ${ }^{97}$

Sin embargo, en estas mismas publicaciones pudieron expresarse las esperanzas depositadas en las nuevas corporaciones, aunque con cierto tono de ironía.

Ya tenemos nuevo ayuntamiento elegido por nosotros mismos. No dudemos ahora terminen muchos males que nos han afligido. Cada un constituyente de él será un verdadero padre de la patria, que desentendiéndose de su interés propio mirará el común como el más principal y sagrado. Debemos dar gracias al Todopoderoso porque nos ha concedido lo que tanto suspiramos, y al augusto congreso labrador de la fuente de nuestra felicidad política. ¡Felices nosotros y nuestros hijos! El comercio, las ciencias, las artes llegarán al más alto grado de perfección. ${ }^{98}$

\section{Conclusiones}

La profusa llegada de noticias a la isla de Cuba de lo que ocurrió en la Península durante este periodo, a pesar de las dificultades en las comunicaciones oficiales, fue posible gracias a la puesta en práctica de todo un servicio de información por parte del capitán general Someruelos, que obtenía noticias fehacientes desde los Estados Unidos. La llegada de prensa desde Europa, rumores y comunicaciones privadas al puerto habanero, así como la posterior publicación de noticias en la propia prenda cubana, facilitó a los ciudadanos cubanos tener conocimiento de los movimientos políticos acontecidos al otro lado del Océano. Esta buena información permitió, entre otras cuestiones, el planteamiento de una propuesta juntista, aunque fallida, en julio de 1808 en La Habana, como otro ejemplo más de lo que estaba ocurriendo casi de manera simultánea en la Península. Noticias como la victoria en Bailén alentaron igualmente la excitación patriótica, así como el establecimiento de la Junta Central, la Elección a Cortes y el envío de representantes provocó el debate sobre la representatividad de las diferentes ciudades de la isla. Sin embargo, la llegada de noticias referentes a cuestiones mercantiles como la libertad de comercio, con claros agravios comparativos con Cádiz y, sobre todo, los debates sobre la esclavitud, acabaron por provocar gran malestar en Cuba. Esta inestabilidad dio lugar a que las reivindicaciones cubanas llegasen a plantear intenciones, al menos, autonomistas. La buena información que se tuvo en Cuba, en especial en La Habana, sobre todo lo que aconteció durante la Guerra de la Independencia, dio como resultado el desarrollo de unas actividades políticas condicionadas por la situación en la Península, mucho más que por los derroteros por los que comenzaron a deslizarse el resto de los dominios españoles en América.

97 NAVARRO GARCÍA, Luis, La Independencia de Cuba, Ed. MAPFRE, Madrid, 1991, p. 63.

98 "Esquila al nuevo ayuntamiento", E.D., La Habana, 1812, BNE, Salón General, HA/ 23554. 